\title{
Entre la justice et la singularité de l'existant: survivances du visage dans la rencontre entre éthique et politique ${ }^{1}$
}

\section{Angela Cristina Salgueiro Marques ${ }^{2}$ \\ Luis Mauro Sá Martino³}

\begin{abstract}
Résumé:
Cet article cherche à réfléchir, à partir des contributions d'Emmanuel Lévinas, sur l'importance d'une articulation entre éthique et justice fondée sur la préservation des survivances du visage dans la rencontre politique et sensible avec l'altérité. Nous soutenons que la transformation constante du champ d'application des règles, lois et normes qui conduisent à la coexistence sociale dépend de notre rencontre éthique avec un Autre qui préserve son étrangeté et qui nous surprend, nous étonne et nous interroge sur notre propre subjectivité, nos actions, besoins et vulnérabilités. Nous montrons comment le visage fonde une relation avec l'Autre qui n'est pas médiatisée par le savoir qui classe et qualifie, mais par l'écoute qui établit une responsabilité guidée par la fraternité et l'hospitalité.
\end{abstract}

Mots-clés: éthique, justice, visage, politique, vulnérabilités.

\section{Introduction}

La contribution d'Emmanuel Lévinas pour l'élaboration d'une conception de justice se trouve dans sa proposition éthique de considérer les sujets, leurs formes de vie et leurs vulnérabilités au-delà des normes, en prenant les singularités qui caractérisent les expériences situées de rencontre avec l'altérité. La dimension éthique de ce rencontre est un thème central de la pensée de Lévinas. Dans certaines de ses œuvres (1980, 1987, 2007, 2014a, 2016), nous trouvons problématisée ce qu'il appelle la «relation sans relation», c'est-à-dire l'interaction originale et asymétrique entre deux interlocuteurs totalement distincts qui ne communiquent pas à travers le discours, mais par le moyen d'une subordination réciproque qui donne origine à une relation transitive dans laquelle l'autre nous échappe toujours. La rencontre éthique qui configure cette «relation sans relation» ne repose pas sur un contrat conflictuel, une dispute argumentative et rationnelle, dans la lutte pour la reconnaissance mutuelle ou même dans la tentative de parvenir à une compréhension symétrique de quelque chose qui se présente

\footnotetext{
${ }^{1}$ Ce travail a été réalisé avec le soutien da la CAPES, du CNPq et da la FAPEMIG.

2 Doutora em Comunicação Social pela UFMG. Professora do Departamento de Pós-Graduação da Universidade Federal de Minas Gerais (UFMG). Instituição : Universidade Federal de Minas Gerais. Brasil. E-mail : angelasalgueiro@gmail.com

${ }^{3}$ Doutor em Ciências Sociais pela PUC-SP. Professor do Programa de Pós-Graduação em Comunicação da Faculdade Cásper Líbero (SP). Instituição: Faculdade Cásper Líbero (SP). Brasil. E-mail : lmsamartino@gmail.com
} 
comme un problème collectif (LIESEN, 2012; ZIELINSKI, 2004). Cette rencontre est possible uniquement car l'émergence du visage nous rend possible le voisinage avec l'autre sans la médiation d'un concept, d'une explication de son inquiétante étrangeté.

Lévinas (1987) souligne que dans la relation intersubjective éthique, l'autre n'est pas nommée, contrôlée ou réduit à un concept, mais invoquée, appelée, obligée de répondre à une demande irréfutable. Selon lui, la représentation et l'appréhension de l'altérité par le concept sont des formes violentes de relation, l'immobilisant et la transformant en une fabrique perverse de conversion de l'autre dans le «soi même». Ainsi, tout contact que se réduit à voir, à appréhender, à connaître, à reconnaître et à comprendre l'altérité est guidée par la nécessité de l'adaptation et de la réduction de la complexité pour transformer l'étrange (l'étrangère) en familier.

La relation primordiale avec autrui cherche cependant à accéder à l'autre dans son étrangeté: elle est faite dans le langage, car elle demande une réponse à l'autre face à son cri, à sa demande irrécusable (ROLANDO, 2001). Mais cette écoute et cette réponse ne sont pas organisées à travers un modèle de conversation ou de dialogue basé sur la symétrie, le consensus ou la reconnaissance mutuelle du statut des interlocuteurs. Loin d'être une interaction ancrée dans l'échange linguistique traduite en signes et discours traversés par des lignes de pouvoir, d'hiérarchie et d'asymétrie, cette relation transitive s'organise sous la forme d'une conversation établie avant le signe.

Il est essentiel de comprendre l'argument selon lequel percevoir l'autre signifie lui répondre dans une attitude de contemplation qui exige une autre temporalité, distendue, décélérée dans laquelle je considère et j'écoute pour accueillir la demande que l'autre m'adresse. En même temps, quand je réponds à son appel, mon identité est aussi constituée: il ne s'agit pas d'une relation de domination ni d'appréhension cognitive ou classement de l'étrangeté des autres (en la réduisant à des typifications et schémas de représentation qui nous permettent de dominer l'univers des choses et les êtres vivants), mais d'une relation de responsabilité (ALVES, 2012; ALVES e GUIGGI, 2012). Répondre à l'autre est devenir responsable de lui, écouter son commandement pour être son otage, sans réduire ou faire disparaitre l'asymétrie et la distance qui empêchent que l'appel soit transformé en une quête de compréhension où de reduction de l'autre à un concept qu'anéantisse la multiplicité de son expérience (SANTOS, 2004, CARRARA, 2010). C'est ce que souhaite conserver Lévinas (2016) dans la rencontre éthique avec l'autre: l'étrangeté qui nécessite la réciprocité et qui reste préservée dans un discours fondateur (d'origine), un mot déconnecté du geste de compréhension. 
Dans ce cadre, la subjectivité est toujours à l'attente et à l'écoute d'une demande adressée à l'autre. Le temps d'attente est aussi le temps de la contemplation et de l'acceptation d'une altérité radicale et étrangère: son arrivée, son appel et sa demande produisent une rupture dans le présent de celui ou celle qu'écoute, l'empêchant de procéder et l'obligeant à élaborer une réponse, a prendre des responsabilités. On pourrait donc dire que c'est l'autre qui apporte le temps futur. «L'autre, en s'approchant, secoue d'abord la corporéité du sujet. A l'approche de l'autre humain commence la constitution de subjectivité » (RIBEIRO, 2015, p.55).

Lévinas (1987) souligne que le temps constitué dans la relation solitaire avec sa propre existence est divisé entre la proximité et le voisinage entre ce sujet ontologiquement seul, et une présence véritablement autre, qui ne se réduit pas à lui-même. Ainsi, «par la venue de l'altérité, l'existence se découvre plurielle et le temps toujours discontinu, toujours renouvelé et recommencé » (RIBEIRO, 2015, p.55). Il est donc essentiel de comprendre l'affirmation selon laquelle percevoir l'autre, c'est l'accueillir et lui répondre dans une attitude d'écoute et de contemplation qui nécessite une autre temporalité : distendue, décélérée, dans laquelle je considère - de façon ample et dépourvue de jugement - la demande vulnérabilité que l'autre m'adresse dans le cadre de nos vulnérabilités exposées.

C'est ce que souhaite conserver Lévinas (2016) dans la rencontre éthique avec l'autre: l'étrangeté qui nécessite la réciprocité, préservée dans un discours fondateur, un mot déconnecté du geste de compréhension. Mais pourquoi est-il si important d'apprécier cette «étrange rencontre» dans laquelle l'individu découvre soi-même en se préoccupant d'attendre l'appel de l'autre? Pourquoi l'étrangeté et la singularité de l'autre devraient-ils, avec la règle du droit, constituer une notion de justice guidée par la fraternité et la responsabilité? Nous proposons, dans ce texte, de réfléchir à ces questions.

\section{Le visage et la communication de la souffrance de l'autre}

Le sentiment de perplexité, d'étonnement face à la présence de l'altérité coexiste, dans la vie quotidienne, avec refus et silence devant l'autre avec lequel on ne veut pas de contact. Le visage, dimension communicative centrale d'une éthique de la responsabilité chez Lévinas, est lié à l'étrangeté de l'autre, à son altérité radicale, inquiétante et irréductible, qui n'est ni conceptualisée ni réduite à la familiarité de la représentation ou du cadre typifiant. Dans la fameuse définition de Lévinas, « le visage parle et me regarde, il m'appelle, il me demande » (1999, p.163). Ainsi, le visage n'est pas réduit à sa manifestation physique (la face 
humaine), mais il fait référence à la transcendance, à l'infini, contre la totalité présente dans la tentative de son appréhension par le concept.

Le visage nous lance un appel qui se distancie de l'hostilité et s'approche de l'hospitalité qu'accueilli tout en posant des questions et en exigeant une réponse. Ainsi, faire l'expérience du visage de l'autre, c'est éprouver un sentiment de responsabilité face à la vulnérabilité, permettant à un individu de se retrouver, de retrouver son identité en répondant à l'appel de l'autre:

[...] il s'agit d'une réponse qu'aucun autre ne peut donner à ma place. En répondant à l'appel de l'autre, je m'affirme comme un sujet unique et irremplaçable. Moi, non interchangeable, je ne suis que dans la mesure où je suis responsable. Je peux remplacer tout le monde, mais personne ne peut me remplacer. (LÉVINAS, 2007, p. 84)

Le visage de l'autre m'affirme (en même temps qu'il m'empêche de faire du mal à celui qui m'interpelle) et rend ma propre existence humaine en me demandant d'écouter et en même temps d'élaborer et d'offrir une réponse. En écoutant et en répondant à l'appel de l'autre, la réponse m'individualise, car elle est unique et ne s'inscrit pas dans le registre de l'universel. Le visage révèle la liberté de l'autre, car sa transcendance continue de nous échapper, quel que soit le degré de conceptualisation ou d'interprétation du visage. Une telle liberté ne renvoie pas à un agent autonome au sens libéral du terme, mais à la relation éthique de considération et d'écoute, générant la possibilité d'acceptation et d'accueil (RAE, 2016, SOUZA et MARQUES, 2016). Mais le visage révèle aussi ma liberté, car pour Lévinas, notre liberté est au service de l'autre, nous le prenons en responsabilité et lui offrons l'hospitalité. À cet égard, le Dire établit une relation et une temporalité entre deux libertés, affirmant l'identité des interlocuteurs. Le Dire permet la transcendance : ne pas être possédée par soimême, investir dans l'exercice d'exposition et de dépossession. Comme argumente Lingis (1987, p.xix):

L'altérité de l'autre n'est pas déterminée, comprise par le soi. L'altérité exerce une force sur le soi, une perturbation dans son émergence infinie et non représentable. Et le moi n'est pas déterminé par l'autre, mais ouvert d'une manière extatique à une transcendance par laquelle il signifie au-delà de lui-même et supporte le poids de la complétude dans sa responsabilité.

Voir l'autre, c'est lui répondre, c'est donner forme à un discours qui possède une force vocative et impérative, structuré comme une réponse et se constituant comme une modalité de reconnaissance qui préserve l'altérité irréductible. Le Dire du visage est une expression, car son sens n'est pas lié à des relations dans lesquelles il se trouve, mais au-delà de lui- 
même: le Dire ne parle pas de quelque chose ou de quelqu'un, il ne s'agit pas d'une information sur quelque chose ou quelqu'un, mais invite quelqu'un à parler à quelqu'un. L'expression a pour objet de défaire la forme sous laquelle l'existant s'expose en tant que thème, dissimulant soi-même (MARCOS, 2010). L'exposition à l'autre définit les identités et rend l'autre important, vital pour l'existence de soi.

\section{"Il faut s'étonner de l'étrangeté de l'autre"}

Dans une interview accordée à Poirié (2007, p.84), Lévinas affirme que l'autre doit toujours garder ses singularités pour ne pas risquer de, lors de son exposition devant le visage que l'interpelle, voir effacé son propre visage par sa réduction à un objet de connaissance : «Situé dans une relation éthique, l'autre homme reste un autre. Ici, c'est précisément l'étrangeté de l'autre, et si l'on peut dire son étrangeté, qui vous lie éthiquement. C'est une banalité, mais il faut y être surpris. » Le visage révèle l'infini et il nous émerveille et nous bouleverse: car en plus d'exposer le différent et l'étrange, il paralyse son pouvoir de tout réduire à un objet qui doit être classé, parfilé sur un cadre d'objets familiers et reconnaissables. Devant l'apparition du visage nous sommes rendus par las surprise parce que

[...] l'infini apparaît comme visage dans la résistance éthique qui paralyse mes pouvoirs et émerge endurcie et absolue du fond des yeux, sans défense dans sa nudité et sa misère. La compréhension de cette misère et de cette faim établit la proximité même de l'Autre. Mais c'est ainsi que l'épiphanie de l'infini est expression et discours (LÉVINAS, 1980, p.178).

L'infini du visage nous étonne parce qu'il résiste à toute tentative d'appréhension et de réduction par la connaissance: il remet en question et refuse de connaître l'autre par le moyen de la représentation, il défie le sujet et son pouvoir de définition, mais accepte la rencontre, la proximité, l'ouverture passive à l'autre.

La proximité se fait par un Dire que porte un sens antérieur aux mots et au signe (le Dit). Ainsi, pour Lévinas (1987, p.119), le langage en tant qu'un contact qui est établi par le Dire « est le langage originel, sans paroles ou propositions, communication pure ». La proximité de l'autre est, en soi même, une signification et ne peut pas être transformé en structure linguistique ou dialogique (relation sans relation que réduit le contact avec l'interlocuteur à l'exercice solitaire et impersonnel de la pensée), car elle est configurée comme un contact. Il est important de préciser que Le Dit désigne un nom et commande le monde, permettant la compréhension des signes à travers le temps, de sorte que la sensibilité 
est un moyen de recevoir des informations venues du monde et de les interpréter, de les classer. Ce que Lévinas a l'intention de faire, c'est de souligner l'irréductibilité du Dire (épiphanie du visage) devant l'imposition constante du Dit, l'irréductibilité de la responsabilité devant la vision ordonnatrice du monde.

À cet égard, « l'immédiateté du sensible est un événement de proximité et pas de connaissance » (LÉVINAS, 1987, p.116). In ne s'agit pas d'une participation des parties prenantes dans une universalité transparente. Il ya dans le discours, dans l'énonciation, « une relation avec le caractère unique situé en dehors du thème dont nous parlons, une singularité qui n'est pas thématisé par le discours, mais que s'approche de lui » (idem). « Un discours dans lequel il y a une complicité libre, une fraternité établie dans mot originel qui dit luimême le Dire » (1987, p.125). Le passage constant de l'intentionnel, de l'objectif à atteindre avec le langage, à l'éthique (contact avec l'unicité insaisissable de l'autre) permet l'émergence du visage qui exige une réponse et qui nous est imposé sans se faire attraper par notre vision. Pourtant, comme nous rappelle Lévinas, « même dans sa subordination à la connaissance, la vision maintient le contact et la proximité. Le visible caresse l'œil, puisque nous voyons et entendons de la même façon que nous pouvons toucher quelque chose ou quelqu'un" (1987, p.118).

Nous nous reconnaissons lorsque nous sommes interpellés par le visage de l'autre: ce n'est pas une soumission, mais un commandement ou un appel fait d'une identité à une autre. Le visage, l'humain, ouvre une fissure sur la peau de l'être, lui rendant otage de l'autre, de l'étranger qui frappe à notre porte (ALFORD, 2004; BURNS, 2008).

Comme nous l'avons déjà mentionné, dans la "relation sans relation" l'autre reste absolument différente, puisque notre dimension "humaine" exige la préservation de la singularité dans la distance: sans cette préservation, la communication éthique perd sa capacité de répondre à la demande de l'autre et à la particularité de ses souffrances (BURNS, 2008). Ainsi, la relation avec le visage ne se fait pas par la connaissance ou le mode de représentation, mais par la sociabilité, par la communication sociale. Chez Lévinas (1995, 2014a), le visage est non seulement la base des relations sociales, mais de l'existence d'un social, mais jamais réductible au social: le visage est la structure intersubjective d'où émergent la politique et la justice. Comme Carrara le souligne (2010, p. 131):

Nous pouvons comprendre la socialité pensée par Lévinas comme une communauté de différents sans que l'universalisation du concept efface les différences. C'est en ce sens que les individualités ne sont jamais perdues dans le tout neutre et anonyme. Les membres d'une telle communauté conservent la capacité d'un mot propre qui les empêche de se perdre dans le langage universel du concept. 
L'ouverture de communication rendue possible par le visage, sous cet aspect, nous place non seulement devant un visage, mais d'une pluralité de visages et des dilemmes liés à la réponse qui doit être élaborée pour chacun d'eux. Cet approche nous permet de penser le champ de la pluralité sociale, puisque chez les tiers "il est possible de passer de l'invisibilité de la relation éthique avec le visage à la visibilité des visages, à l'être ensemble dans un lieu" (CARRARA, 2010, p.90). L'émergence du "tiers" marque l'ouverture d'un passage entre l'éthique et la politique:

Chez Lévinas, avec le Tiers et le dépassement de la dyade, nous sommes placés dans l'ordre de la calculabilité, de la justice distributive, des lois approuvées par la majorité et donc dans le domaine du politique, compris comme un ensemble de règles formalisables. Bien que la dimension sociale du politique ne nie pas l'éthique et sa prétention, il n'est toujours pas facile de dire comment la revendication éthique survit dans le domaine social et politique (BUTLER, 2017, p.225).

La justice se configure ainsi en dehors d'une relation de dualité: elle généralise, initie une égalité (différente de l'asymétrie radicale qui se présente dans la relation sans relation), compare, organise et réduit les singularités et les distinctions à une normativité qui n'accepte pas facilement les exceptions. Il est extrêmement difficile, mais surtout pas impossible, de faire dialoguer l'éthique et la justice. Comme le souligne Carvalho (2018, p. 42), il est nécessaire de penser que le droit et la justice sont étroitement liés à la dignité humaine, puisque la dignité est une construction et une conquête réciproque et non un privilège convenu : «Le chemin de passage qui mène à la dignité est le même qui permet le droit de rencontrer une justice qui est toujours en élaboration dans sa juste (in)détermination. "

Selon Ribeiro (2018), la justice doit être la justice du visage, c'est-à-dire qu'elle doit chercher à se réaffirmer dans son mouvement de renouvellement constant par l'éthique, par le dérangement causé par la présence d'une altérité radicale.

Comment la justice peut-elle permettre une certaine ouverture au souffle renouvelé du Dire que démenti pour la reconstruire à nouveau? [...] Nous croyons que, pour que la justice puisse se renouveler, il faudrait dans un certain sens, et malgré elle, l'irruption de la singularité des individus et de leurs souffrances que se produit dans l'inexorabilité de la rencontre non désirée avec le visage. [...] Pour nous, la persistance de l'être qui comporte la justice est malgré elle, se rapprochant ainsi du malgré soi du «moi» persistant dans son être devant l'autre. En tant qu'être, la justice cherche à persister pour ne pas changer, en restant sous la forme du Dit, du droit. (RIBEIRO, 2018, p.122-123).

Ce sont les passages entre le Dit et le Dire qui rendent la justice perméable à la diversité, aux micro-politiques quotidiennes et aux vulnérabilités situées qui nécessitent la proposition de politiques publiques tenant compte de l'intersectionnalité qui donne corps aux 
revendications et aux expériences dans la quête de la promotion de conditions concrètes de promotion de la justice.

\section{Le visage entre l'éthique et la justice}

Selon Lévinas (1987, 2007, 1980), l'autre ne se trouve pas en tant qu'un être objectifiable, quelqu'un à être contrôlé ou possédé. L'autre se manifeste devant nous comme visage: c'est dans la rencontre avec le visage de l'autre que se révèle l'infinie altérité de l'autre. Il établie une relation de discours: l'autre n'est pas seulement nommée, il est aussi invoquée. Le visage de l'autre n'est pas capté par la vue, car il ne peut être saisi par la représentation dans le langage. La vision ne prévaut pas dans la rencontre avec le visage, car elle est une forme de relation violente avec l'autre: elle porte la menace de transformer l'autre en "soimême" une fois que l'œil appréhende par la classification et la connaissance. La vision ne rend pas justice à l'autre (ROBBINS, 1991). C'est ainsi que le visage se manifeste à l'écoute, en tant qu'une voix, un appel, un cri.

Le passage de la vision à la responsabilité, de la représentation à la voix, implique une rupture: l'autre n'est pas appréhendée par la vue, mais par l'écoute. La rencontre éthique avec l'autre interrompt la tendance du self à concevoir le monde comme un espace de pouvoir dans lequel il agit souverainement. L'exposition interrompt le jeu de la réduction de l'autre à "soi-même" et nous libère de notre petit monde de plaisirs et de soucis, nous rendant en otage. L'éthique de la rencontre est guidée par une remise en question de ma liberté, qui n'est concrétisée qu'au service de l'autre. Cependant, la liberté n'est pas la servitude, mais la compréhension qu'en servant l'autre, je m'ouvre à l'infini, à l'absolument autre (ALFORD, 2004; MARCONDES FILHO, 2007).

Ainsi, la relation primordiale avec le visage de l'autre est le discours: une conversation qui propose le monde, mais garde la distance d'une séparation infinie. Le paradoxe de cette relation est que la distance détruit la relation et que la relation détruit la distance. Le visage parle, lance son appel et instaure une situation concrète dans laquelle la souffrance de l'autre nous émeut, nous retire de nous-mêmes et exige une réponse. Il est important de souligner que la souffrance des autres est révélée dans un contexte particulier de rencontre que nous demande un changement, une réorientation (temporelle, spatiale et corporelle) vers la recherche des conditions pour aller à leur aide. L'autre qui me demande une écoute souffre et existe dans un contexte spécifique, marqué par des vulnérabilités changeables et que se modifient selon les ressources (matérielles, affectives, culturelles, politiques) dont nous avons 
accès pour produire des dispositifs, des arrangements qui peuvent nous conduire à la réponse souhaitée (BURNS, 2008; SOUZA et al., 2016 ; MARQUES ; SOUZA, 2018).

Selon Burns (2008), la souffrance et la vulnérabilité de l'autre ne sont pas simplement des faits objectifs que le sujet identifie comme tels. Au lieu de cela, la souffrance impose à celui ou celle qui en est témoin l'obligation de justifier cet état injuste. Le visage a donc une autorité éthique unique pour obtenir une réponse du sujet. C'est pourquoi l'autre me fait face comme un suppliant qui n'a rien et en même temps comme le maître qui me commande d'en haut. En tant que témoin de la souffrance et en tant qu'otage à cause de ce témoignage, le sujet assume une responsabilité unique vis-à-vis de l'autre et avant son propre statut «privilégié» ou sa fermeture dans l'égoïsme et le silence.

Il y a donc un contexte d'action dans lequel nous sommes fréquemment pris par la volonté de substituer physiquement l'autre dans sa souffrance. En ce sens, Levinas (2007) souligne que la responsabilité exige un engagement charnel, puisque devant la douleur de l'autre matérialisée sur son corps, nous interrompons notre isolement et nous allons au secours des autres, parfois au point d'affliger notre corps avec des expiations au nom de celui ou celle qu'à ce moment-là, est perçu(e) comme notre invité, qui se bénéficie de notre hospitalité. Ainsi, le visage n'e se dissocie pas totalement d'un corps et des gestes qui rendent possible l'affectation de nos sens par les blessures et afflictions de l'autre.

Butler (2015) nous offre également des indices sur les situations dans lesquelles les demandes sont produites et les formes sont assumées par le Dire du visage. La clameur que l'autre me dirige nécessite une médiation, un véhicule qui abrite les sens et que les transportent:

Les revendications qui me sont faites sont établies (si jamais elles le sont) par les sens, qui sont en partie façonnées par les différentes formes de médias: l'organisation sociale du son et de la voix, de l'image et du texte, du geste de toucher et de l'odorat. Si les revendications de l'autre me sont adressées, elles doivent faire l'objet d'une médiation, ce qui signifie que notre propre capacité à réagir sans violence, à agir contre un acte de violence ou à préférer la non-action à la provocation violente dépend des cadres dans lesquels le monde mous est donné et le domaine d'apparence est circonscrit. (BUTLER, 2015, p.252)

Butler souligne, en dialogue avec la pensée de Lévinas, les difficultés à établir des articulations entre la rencontre éthique avec l'altérité et l'élaboration de la justice dans les processus politiques. Elle mentionne qu'il ne s'agit pas de choisir l'une au détriment de l'autre, mais de réfléchir aux conditions dans lesquelles nous devenons réceptifs et accueillants envers les autres, donnant lieu à un cadre situationnel et moral qui guide les décisions et les normes collectives. À cet égard, elle souligne qu'une tentative de répondre et d'être 
responsable face à l'autre au sein d'un processus politique doit prendre en compte «une relationnalité ex-tactique, une manière de se dépasser, de se déposséder de soi-même dans la relation avec un autre qui m'appelle et que me sollicite" (2017, p. 18).

Elle affirme (2017, p. 27) être à la recherche de moyens de formuler une éthique qui commence par la question des conditions de réception des messages, des injonctions ou des commandes provenant d'une autre sphère discursive, sphère mal intégrée à la nôtre. À cet égard, l'expression du visage (son Dire) ne peut être entendue qu'à condition qu'elle soit traduite "[...] dans les circonstances concrètes dans lesquelles quelqu'un vit, dans la situation dotée du sens historique et géographique, dans les scènes de violence qui imprègne la vie quotidienne".

Il est donc possible de parler de l'existence d'une éthique communicative dans la rencontre asymétrique avec une altérité radicale. Pourtant il s'agit d'une éthique qui ne repose pas sur des principes universels (comme l'éthique habermasienne du discours), mais qui offre la structure intersubjective de "relation sans relation" d'où peut émerger le besoin de règles et de lois morales (BURNS, 2008). Dans cette éthique communicative, la personne qui souffre me défie à partir de sa souffrance et exerce son autorité sur moi.

Le visage de celui pour qui j'assume la responsabilité m'interpelle: je lui dois une justification de l'inégalité qui réitère sa vulnérabilité et sa précarité, mais son visage transcende le contexte de l'action. Comment gérer cette altérité infinie et abstraite dans la résolution de problèmes moraux? Il faut d'abord considérer la complémentarité entre deux dimensions de la notion de visage: la transcendance et le Dire. Le Dire du visage, comme nous l'avons vu, établit un contexte possible de rencontre dans le langage antérieure au signe (LÉVINAS, 1999, p.163). La singularité du visage et sa transcendance se produisent dans un contexte qui lui donne une figure. Dans le Dit, il y a l'articulation d'un monde donné, déjà institué. Contrairement, le Dire comporte la trace de l'altérité.

La force du Dit, du code d'homogénéisation de la loi, arrache le visage de ce contexte et lui imposent une violence énorme, le défigurant. Lévinas (2014a) insiste sur cette tension, mais aussi sur la nécessité de complémentarité entre figure et visage défiguré, entre le Dire et le Dit, surtout par rapport aux dimensions communicatives et corporelles de la responsabilité éthique et de la justice. Sa critique de la manière dont la justice peut conduire au totalitarisme est directement liée à un appel à la préservation de l'unicité et de la singularité du visage. Selon lui, la justice réduit la rencontre avec l'altérité à la comparaison et à la connaissance, empêchant ainsi le lien de responsabilité de se constituer. Si la justice reste aveugle au visage, je cesse de craindre pour la mort de l'autre, car il ne me concerne plus, ne 
me rend plus responsable de lui. C'est pourquoi, pour Lévinas, la normativité éthique commence dans la rencontre avec l'altérité, avec le visage.

Les hommes autour de moi sont nombreux! D'où le problème: qui est mon voisin? Problème inévitable de la justice. La nécessité de comparer l'incomparable, de connaître les hommes; d'où leur apparition en tant que formes plastiques de figures visibles et, dans un sens, "défigurées": en tant que groupe dont l'unicité du visage est déchirée d'un contexte, source de mon obligation devant les autres hommes; une source à laquelle se réfère, après tout, la même quête de justice et dont l'oubli risque de se transformer en calcul purement politique - et même en abus totalitaires - le travail sublime et difficile de la justice (LÉVINAS, 2014a, 28-29)

Nous avons vu que le visage regarde et appelle par le langage. Par conséquent, trouver un visage qui parle et donner une voix au visage revient à lui donner une figure. Mais encore une fois, le rapport du discours à l'autre n'est pas la confrontation de deux figures, mais l'accès à l'autre dans son étrangeté par la parole. Mais pour maintenir cette étrangeté, il faut la non-réciprocité et la non-compréhension. Je reçois un mot que je ne peux pas comprendre, mais je dois y répondre. Ce dialogue est un discours sans "pouvoir", car l'autre a interrompu mon pouvoir et mon contrôle (ROBBINS, 1991 ; GUIMARÃES, LIMA, 2013).

Mais que faisons-nous face à la nécessité de répondre à l'appel des différents visages qui se manifestent devant nous? Il ne serait pas possible de répondre à tout le monde de la même manière, sans laisser de privilégier les uns et de condamner les autres. Lévinas (2014a) indique que la production de cette réponse doit combiner la "relation sans relation" avec le visage et la définition de lois fondant une justice orientée par les singularités des revendications et non par le privilège d'une rationalité régie exclusivement par un ordre objectif qui préserve et protège la liberté de tous (ou de certaines personnes). Il soutient que la justice peut être violente en ne reconnaissant pas les individus dans leur précarité et leurs vulnérabilités (le sens du visage nu de l'autre), en les traitant de la même manière et en soumettant l'individualité et la particularité à des catégories universelles. (ALFORD, 2004; SANTOS, 2004).

La manière de répondre aux demandes multiples des différents visages qui se présentent à nous ne peut pas être résolue uniquement sur le plan éthique, mais nécessite une décision prise dans le domaine de la moralité et de la justice. La politique découle de cette relation éthique et communicationnelle qui la précède et implique la réception, la rencontre et la responsabilité. Cependant, la politique de Lévinas ne se traduit pas dans la relation dialectique et discursive de l'argumentation. La relation établie par la rencontre avec le visage est asymétrique et ne se réduit pas à une totalité. C'est précisément l'éthique qui découle de l'écoute de la voix du visage qui peut empêcher le totalitarisme et l'universalisation: "chaque 
fois que la politique s'éloigne des exigences de la responsabilité face à l'autre elle perd sa capacité de produire la justice er l'hospitalité " (CARRARA, 2010, p. 92).

Si le visage d'un sujet ne diffère pas d'un autre, au sens moral du terme, tous mériteraient également attention (BURNS, 2008, p. 316). Cependant, Lévinas nous dit que la rencontre avec le visage est situationnelle et, bien qu'il nous échappe constamment, il lui faut un moment pour « faire figure », pour apparaitre et pour nous adresser, matériellement, son appel. Ainsi la réponse que nous adressons à l'autre est singulière à force de la demande aussi unique qui nous saisie. L'obligation de responsabilité est donc toujours distincte devant chaque visage qui se présente à nous. Comme le souligne Butler:

En termes philosophiques, Lévinas décrit une scène éthique dans laquelle nous sommes obligés, dans la plupart des situations, de préserver la vie de l'autre - obligés par l'altérité que nous y trouvons. Sous un regard plus attentif, on s'aperçoit que cette scène, qui semblait nous contraindre universellement, est limitée en termes culturels et géographiques. L'obligation éthique envers le visage de l'autre n'est pas une obligation que l'on peut ressentir par rapport à chaque visage. À ce propos, Derrida a déclaré que s'il devait réagir à tous les visages, il deviendrait inévitablement irresponsable. Si cela est vrai, l'exigence éthique ne précède pas les notions d'autonomie culturelle, mais est encadrée et limitée à l'avance par certaines notions de culture, d'ethnie et de religion. (BUTLER, 2017, p.47)

En ce sens, Lévinas $(1980,2007)$ propose que, face à la multiplicité des visages qui nous interpellent, le geste de décider à qui répondre et de quelle manière est un geste politique qui implique la norme et le code moral et éthique, mais requiert également la fraternité, l'amitié et l'hospitalité. L'élaboration de la réponse dépende des liens dans lesquels la responsabilité en tant que relation éthique ne signifie pas seulement assumer le fardeau de l'autre ou lui aider à supporter les épreuves de l'existence, mais aussi porte quelque chose de spontané, d'intuitif, de sorte que l'expérience du visage de l'autre et la demande qu'elle nous adresse est positive et enrichissante puisque "c'est à partir de l'existence de l'autre que ma propre existence devient humaine" (LÉVINAS, 1995, p.167).

Lévinas (1980) souhaite préserver la rencontre éthique capable de configurer l'identité des interlocuteurs, en tenant compte de l'importance que la vie d'un sujet, dans sa vulnérabilité, a pour l'autre: "il est très important pour moi que la justice puisse couler, en prenant sa source à partir de la prééminence de l'autre "(LÉVINAS, 2014a, p. 35), et en évitant que la politique mette en place une forme de jugement moral qui tue et efface le visage, les visages qui se lèvent devant nous. Il ne nie pas la nécessité des lois et des codes de justice, mais il nous rappelle qu'avant de nous mettre au service d'une raison impersonnelle, deux libertés différentes doivent s'approcher et se rapporter sans la médiation de la raison: une relation de commandement sans la tyrannie, non limitée par le devoir d'obéissance à la loi 
universelle, et pourtant une condition indispensable à l'institution de la loi. Si jamais nous n'approchons des autres dans leur unicité et singularité, si nous ne regardons jamais dans les yeux de l'autre qui demande un positionnement, la domination trouve sa place et nous cessons de craindre pour la mort d'un autre qui ne signifie donc rien pour nous. Le visage défiguré ne nous empêche pas de tuer.

Bulter (2017, p. 64) pose une série de questions qui semblent constituer un programme de recherche à explorer dans ses propres études sur une éthique de la non-violence et de la dépossession que considère le visage comme point de départ pour la reconnaissance:

Le visage de l'autre secoue donc tous les formalismes, car le formalisme me ferait traiter tous les autres avec la même importance et, par conséquent, personne ne me réclamerait jamais. Mais pouvons-nous vraiment nous passer de tous les formalismes? Et si nous ne pouvons pas nous passer de tous les formalismes, alors comment pouvons-nous penser au visage par rapport à de telles normes politiques? Le visage doit-il être singulier à tout moment ou peut-il s'étendre à la pluralité? $\mathrm{Si}$ le visage n'est pas nécessairement un visage humain - ce peut être un son ou un cri - et s'il n'est pas réduit au visage d'une seule personne, peut-il être généralisé à toute personne, dans la mesure où il me concerne également? Est-ce une rupture dans la manière dont nous pensons à la pluralité ou représenteraitelle une entrée dans l'éthique précisément dans la formulation de la pluralité elle-même? Le visage peut-il servir d'injonction contre la violence dirigée contre chaque individu, y compris ceux dont les visages, littéralement, je ne sais pas? Est-il possible de dériver une politique de nonviolence de l'injonction de lévinasian, et sera-t-il possible de répondre aux visages de cette foule?

La fraternité, en tant que responsabilité devant tous, fait en sorte que la justice puisse découler de l'inquiétude et de la non-indifférence engendrées par la proximité de l'étranger à qui nous offrons l'hospitalité. Elle se situe entre la justice normative et rationnelle et l'injustice, en faisant "appel à la sagesse du moi dont les possibilités ne contiennent probablement aucun principe établi à priori" (LÉVINAS, 2014a, p. 35). Nous voyons ici une importante dimension esthétique et éthique de la politique chez Lévinas: pour lui, la source de la justice n'est pas uniquement dans l'ensemble des normes et valeurs qui dirigent les institutions, mais surtout dans la sagesse, les expériences singulières qui ne sont pas capturés et cartographiés par la règle, qui ont la possibilité de renouveler les passages entre le Dit et le Dire, et ainsi de nourrir des nouvelles formes de vie en dehors des réglementations disciplinaires, actualisant le devenir d'un commun que, provisoirement, la communication cherche à construire.

Le visage peut-il survivre dans le domaine de la politique normative et de la justice? Lévinas prétend que oui, parce que le visage et sa directive humanisant, son impératif de nous rendre sensibles à la vulnérabilité de l'autre et son exigence de dépossession, nous indiquent 
que des choix politiques équitables sont faites entre la condamnation et l'écoute du visage, entre clarté de la règle et l'opacité de l'inquiétant.

\title{
Entre a justiça e a singularidade do que existe: sobrevivência do rosto no encontro entre ética e política
}

\section{Resumo:}

Esse artigo procura, a partir das contribuições feitas por Emmanuel Lévinas, refletir acerca da importância de uma articulação entre a ética e a justiça a partir da preservação das sobrevivências do rosto no encontro político e sensível com a alteridade. Argumentamos que a transformação constante do escopo de regras, leis e normas que conduzem a convivência social depende de nosso encontro ético com um Outro que preserva sua estranheza e que, por isso mesmo, nos surpreende, nos espanta e nos indaga reciprocamente sobre nossa própria subjetividade, nossas ações, necessidades e vulnerabilidades. Mostramos como o rosto funda uma relação com o Outro que não é mediada pelo conhecimento que tudo classifica, mas pela escuta que instaura uma responsabilidade guiada pela fraternidade e pelo acolhimento.

Palavras-chave : ética, justiça, rosto, política, vulnerabilidades.

\section{Between justice and the singularity of what exists: survival of the face in the encounter between ethics and politics}

\begin{abstract}
This article seeks, based on the contributions made by Emmanuel Lévinas, to reflect on the importance of an articulation between ethics and justice based on the preservation of face survivals in the political and sensitive encounter with otherness. We argue that the constant transformation of the scope of rules, laws and norms that lead to social coexistence depends on our ethical encounter with an Other that preserves his strangeness and that, for this very reason, surprises us, amazes us and asks us reciprocally about our own subjectivity, our actions, needs and vulnerabilities. We show how the face founds a relationship with the Other that is not mediated by the knowledge that classifies everything, but by a listening that establishes a responsibility guided by fraternity and acceptance.
\end{abstract}

Keywords: ethics, justice, face, politics, vulnerabilities. 


\section{Références}

ALFORD, C. Fred. Lévinas and Political Theory. Political Theory, v.32, n.2, 2004, p.146171.

ALVES, Marcos Alexandre. Ética como filosofia primeira: sabedoria da paz/palavra em Lévinas. Acta Scientiarum, v. 34, n. 1, 2012, p.49-58.

ALVES, Marcos Alexandre; GHIGGI, Gomercindo. Pedagogia da Alteridade: o ensino como condição ético-crítica do saber em Lévinas. Educação e Sociedade, Campinas, v. 33, n. 119, p. 577-591, abr.-jun. 2012, p. 577-591.

BURNS, Lawrence. Identifying Concrete Ethical Demands in the Face of the Abstract Other: Emmanuel Levinas' Pragmatic Ethics. Philosophy and Social Criticism, v. 34, n.3, 2008, p.315-335.

BUTLER, Judith. Caminhos Divergentes. São Paulo: Boitempo, 2017.

BUTLER, Judith. Quadros de guerra: quando a vida é passível de luto? Rio de Janeiro: Civilização Brasileira, 2015.

CARRARA, Ozanan. Lévinas: do sujeito ético a sujeito político. São Paulo: Ideias \& Letras, 2010.

CARVALHO, Felipe Rodolfo de. "Dignidade Humana e Ordem Jurídica do desejo". In: RIBEIRO JÚNIOR, Nilo et al. (orgs). Amor e Justiça em Lévinas. São Paulo: Perspectiva, 2018, p.27-42.

GUIMARÃES, César.; LIMA, Cristiane. Ser-em-comum: Notas para uma aproximação entre a Ética de Lévinas e a Episteme comunicacional. Eco-Pós, v. 16, n. 1, 2013, p. 74-89.

LÉVINAS, Emmanuel. A violência do rosto. São Paulo: Loyola, 2014a.

LÉVINAS, Emmanuel. Descobrindo a existência com Husserl e Heidegger. Lisboa: Instituto Piaget, 2014b.

LÉVINAS, Emmanuel. Alterité et transcendence. Paris: Fata Morgana, 1995.

LÉVINAS, Emmanuel. Alterity and transcendence. New York: Columbia University Press, 1999.

LÉVINAS, Emmanuel. Autrement qu'être. Paris: Livre de Poche, 2016.

LÉVINAS, Emmanuel. Entre nós. Petrópolis: Vozes, 2010.

LÉVINAS, Emmanuel. Ética e Infinito. Lisboa: Ed. 70, 2007.

LÉVINAS, Emmanuel. Totalidade e Infinito. Lisboa: Ed. 70, 1980.

LÉVINAS, Emmanuel. Collected Philosophical Papers, Trad. Alphonso Lingis, Dordrecht: Martinus Nijhoff Publishers, 1987.

LIESEN, Mauricio. Por uma comunicação como acolhimento e impossibilidade. Intexto, 
Porto Alegre, UFRGS, n.26, 2012, p.81-97.

MARCONDES FILHO, Ciro. O outro como um mistério e o feminino como a alteridade absoluta. Sobre a recuperação do face-a-face na comunicação em Emmanuel Lévinas. Matrizes, no. 1, Vol. 1, Outubro 2007, p. 55-74.

MARCOS, M. L. Comunicação, experiência e a questão do reconhecimento. Entrevista a Reges Schwaab. Revista Brasileira de Ciências da Comunicação, São Paulo, v.33, n.2, p. 241-251, jul./dez. 2010, p. 241-263.

MARQUES, A. C. S.; SOUZA, F. C. V. Politics and aesthetics in Rancière and Lévinas: scene of dissensus, face and constitution of the political subject. Kriterion, v. 139, p. 7-34, 2018.

MENEZES, Magali Mendes. O pensamento de Emmanuel Lévinas: uma filosofia aberta ao feminino. Estudos Feministas, Florianópolis,v.16, n.1, 2008, p. 13-30.

POIRIÉ, François. Emmanuel Lévinas: Ensaio e Entrevistas. São Paulo: Perspectiva, 2007.

ROBBINS, Jill. Visage, figure: reading Lévinas Totality and infinity. Yale French Studies, n.79, 1991, p.135-149.

RAE, Gavin. The political significance of the Face: Deleuze's Critique of Lévinas. Critical Horizons, v.17, n.3-4, 2016, p.279-303.

RIBEIRO, Luciane. A Subjetividade e o Outro: ética da responsabilidade em Emmanuel Levinas. 1. ed. São Paulo: Ideias\&Letras, 2015.

RIBEIRO, Leonardo. “Amor e Justiça em Lévinas”. In: RIBEIRO JÚNIOR, Nilo et al. (orgs). Amor e Justiça em Lévinas. São Paulo: Perspectiva, 2018, p.115-130.

ROLANDO, R. Emmanuel Lévinas: para uma sociedade sem tiranias. Educação \& Sociedade, ano XXII, n. 76, 2001, p. 76-94.

SANTOS, M. L. L. Q. Subjetividade como responsabilidade em Lévinas: quando a alteridade atravessa o sujeito. Recife: UFPE, 2004 (Dissertação de Mestrado).

SOUZA, Frederico Vieira.; MARQUES, Angela. Rosto e cena de dissenso: aspectos éticos, estéticos e comunicacionais de constituição do sujeito político. Questões Transversais, vol. 4, no. 7, janeiro-junho 2016, pp. 17-28.

SOUZA, Frederico; COELHO, Tamires; MARQUES, Angela. O rosto na imagem, a imagem sem rosto. Trabalho apresentado ao Grupo de Trabalho Comunicação e Experiência Estética do $X X V$ Encontro Anual da Compós, na Universidade Federal de Goiás, Goiânia, de 7 a 10 
de junho de 2016.

ZIELINSKI, A. Lévinas: la responsabilité est sans pourquoi. Paris: PUF, 2004.

Trabalho recebildo em 06 de fevereiro de 2019

Aceito em 12 de fevereiro de 2019 